\title{
On moment closures for population dynamics in continuous space
}

\author{
David J. Murrell ${ }^{\mathrm{a}, *}$, Ulf Dieckmann ${ }^{\mathrm{b}}$, Richard Law ${ }^{\mathrm{c}}$ \\ ${ }^{a}$ NERC Centre for Population Biology, Imperial College at Silwood Park, Ascot, Berkshire SL5 7PY, UK \\ b Adaptive Dynamics Network, International Institute for Applied Systems Analysis, A-2361 Laxenburg, Austria \\ ${ }^{\mathrm{c}}$ Biology Department, York University, PO Box 373, York YO10 $5 \mathrm{YW}$, UK
}

Received 14 August 2003; received in revised form 8 April 2004; accepted 9 April 2004

\begin{abstract}
A first-order moment closure, the mean-field assumption that organisms encounter one another in proportion to their spatial average densities, lies at the heart of much theoretical ecology. This assumption ignores all spatial information and, at the very least, needs to be replaced by a second-order closure to gain understanding of ecological dynamics in spatially structured populations. We describe a number of conditions that a second-order closure should satisfy and use these conditions to evaluate some closures currently available in the literature. Two conditions are particularly helpful in discriminating among the alternatives: that the closure should be positive, and that the dynamics should be unaltered when identical individuals are given different labels. On this basis, a class of closures we refer to as 'power-2' turns out to provide a good compromise between positivity and dynamical invariance under relabelling.
\end{abstract}

(C) 2004 Elsevier Ltd. All rights reserved.

Keywords: Moment closure; Population dynamics; Spatial

\section{Introduction}

The topic of moment closures for population dynamics sounds at first sight a somewhat esoteric subject for ecologists. Yet the subject provides a key to unlock the formal structure of population dynamics in continuous space (Bolker, 1999; Bolker and Pacala, 1997, 1999; Bolker et al., 2000; Dieckmann and Law, 2000; Law and Dieckmann, 2000a, b; Law et al., 2003a; Murrell and Law, 2000, 2003; Pacala and Levin, 1997). Dynamics in discrete space also need moment closures (Boots and Sasaki, 1999; Ellner, 2001; Filipe and Gibson, 2001; Filipe and Maule, 2003; Harada et al., 1995; Matsuda et al., 1992; Rand, 1999; Sato and Iwasa, 2000; Sato et al., 1994; van Baalen, 2000); therefore, many of the results presented in this paper are expected also to be of direct relevance for such models.

The reason why moment closures are important becomes evident when dynamical systems of spatial moments are derived from underlying stochastic models

*Corresponding author. Tel.: +44-20-759-42471; fax: +44-1344873173.

E-mail address: d.murrell@ic.ac.uk (D.J. Murrell). of individual births, deaths, and movements (Bolker and Pacala, 1997; Dieckmann and Law, 2000; Rand 1999; Sato et al., 1994). The dynamics of the first spatial moment (average over space of population density) turn out to have flux terms involving the second moment (a spatial covariance or pair density; see Section 2.1). The dynamics of the second spatial moment have flux terms involving the third moment (a density of triplets; see Section 2.1). And so on. The dynamics of each spatial moment are coupled to the next spatial moment in the hierarchy.

To close the dynamical system, the next spatial moment in the hierarchy must, at some stage, be replaced with an expression containing only lower-order moments. This expression is a moment closure. The simplest closure, at order one, is used whenever the second moment is replaced by a product of two first moments (mean densities); this is the mean-field assumption that individuals encounter one another according to their average densities over space, like molecules in an ideal gas. The mean-field assumption has certainly served ecology well for the last 80 years, but it does ignore all spatial information. Arguably ecological theory now needs a closure that operates at 
least at order two, to deal with the spatial structure increasingly recognized as important in studies of ecological dynamics (Dieckmann et al., 2000; Hubbell et al., 2001; Keeling, 1999; Norris et al., 2001; Stoll and Prati, 2001; Tilman and Karieva, 1997). With such a closure, we would be in a position to describe crucial aspects of spatial patterns, including, in particular, the spatial aggregation and segregation of organisms.

A moment closure is an assumption. But there are certain qualities that, desirably, a closure should have, if it is to be a reasonable replacement for a higher-order moment. In this paper we describe all the qualities we are aware of and, on this basis, evaluate a number of closures that have been suggested in the literature.

\section{Preliminaries}

\subsection{A spatial pattern and its moments}

For notational simplicity, we consider a single-species population; the extension to a multispecies spatial pattern is straightforward (Dieckmann and Law, 2000; Law and Dieckmann, 2000a). The population lives in a continuous spatial region of size $A$; for convenience, the space is taken as two-dimensional and large enough for edge effects to be negligible. An individual $l$ at location $x_{l}=\left(x_{1 l}, x_{2 l}\right)$ is denoted by a Dirac delta function $\delta_{x_{l}}(x)$ with a peak at $x_{l}$ and zero elsewhere; the spatial pattern of individuals $p(x)$ is then the sum of all the delta functions (Dieckmann and Law, 2000; pp. 416-417).

The first spatial moment $N(p)$ is simply the mean density, familiar as the state variable of mean-field population dynamics,

$N(p)=\frac{1}{A} \int p(x) \mathrm{d} x$.

This first moment of course does not hold any information on how individuals are distributed across space.

To measure second-order spatial structure, we use the second moment,

$C(\xi, p)=\frac{1}{A} \int p(x)\left[p(x+\xi)-\delta_{x}(x+\xi)\right] \mathrm{d} x$.

This measures the density of pairs with a spatial displacement $\xi=\left(\xi_{1}, \xi_{2}\right)$ from the first to the second individual in the pair. Such a pair density is closely related to the central spatial covariance, spatial autocorrelation, and semi-variance measures more commonly used in the ecological literature (see Burrough, 1995; Dale, 1999; Wiegand and Moloney 2004); in the context of moment dynamics, the pair density has the advantage of being a particularly simple and immediately intuitive measure of second-order spatial structure. The Dirac delta function $\delta_{x}(x+\xi)$ removes

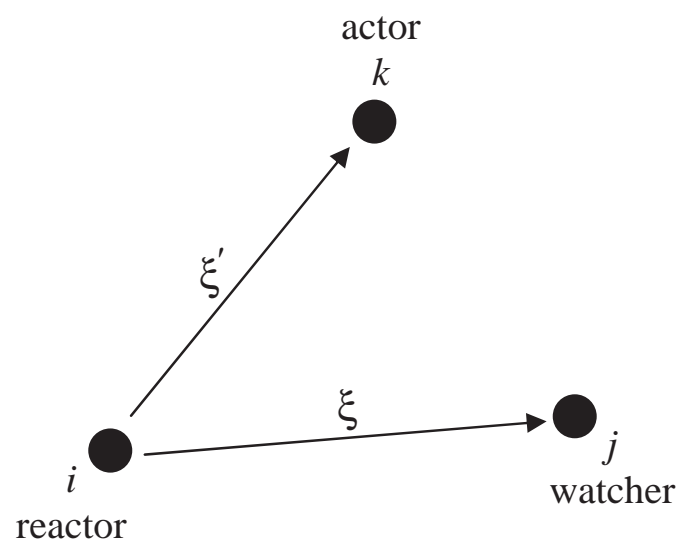

Fig. 1. Geometry of a triplet of three individuals labelled as in the text. The third moment describes the density $T\left(\xi, \xi^{\prime}\right)$ of such triplets, and closures of the third moment predict this density in terms of the densities $N$ and $C$ of singlets and pairs, respectively. In a purely static and descriptive context the corners of the triplet are interchangeable, whereas in the context of dynamics the corners assume different roles and, accordingly, are labelled reactor, watcher, and actor (see Section 4).

the self-pair terms that arise when the spatial displacement $\xi$ is zero.

The first and second spatial moments above are just the first two terms in a hierarchy of moments. The next term,

$$
\begin{aligned}
T\left(\xi, \xi^{\prime}, p\right)= & \frac{1}{A} \int p(x)\left[p(x+\xi)-\delta_{x}(x+\xi)\right]\left[p\left(x+\xi^{\prime}\right)\right. \\
& \left.-\delta_{x}\left(x+\xi^{\prime}\right)-\delta_{x+\xi}\left(x+\xi^{\prime}\right)\right] \mathrm{d} x
\end{aligned}
$$

is the density of triplets with the triplet's second individual displaced from the first by $\xi=\left(\xi_{1}, \xi_{2}\right)$, and the third by $\xi^{\prime}=\left(\xi_{1}^{\prime}, \xi_{2}^{\prime}\right)$, as shown in Fig. 1. The delta functions again remove self-pairs that would otherwise arise when $\xi$, or $\xi^{\prime}$, or both are zero. The extension to higher-order moments continues in the same way.

\subsection{Dynamical system of spatial moments}

The spatial pattern and the moments above deal with the state of a single population at an instant in time, $t$. In reality, the spatial pattern changes over time as individuals are born, die, and move around. Moreover, because births, deaths, and movements are stochastic events, rerunning the stochastic process from the same starting conditions would not give rise to exactly the same pattern at $t$. The spatial moments at $t$ thus have their own probability distributions, and we can describe the population dynamics in terms of the statistics of these distributions. To emphasize the distinction between the spatial moments of a particular spatial pattern above, and the dynamics of a moment obtained by averaging over many realizations of a stochastic process 
below, the argument $p$ is only used in the former but not in the latter case.

For simplicity, we develop ideas in the context of a spatial dynamical system of just one species, a logisticlike model of population growth with births, deaths, and movement at birth (Law et al., 2003a). The dynamical system has the first and second moments as state variables, and has been derived analytically from a stochastic, individual-based process of births, deaths and movements in a continuous space (Dieckmann and Law, 2000; see also a related derivation by Bolker and Pacala, 1997). Dynamics of the first moment are given by

$\dot{N}=(b-d) N-d^{\prime} \int w(\xi) C(\xi) \mathrm{d} \xi$.

In this equation, individuals have constant intrinsic rates of birth and death, $b$ and $d$, respectively. In addition, there is a death rate dependent on the density of neighbouring individuals with terms as follows. First $w(\xi)$ is a competition kernel, normalized so that the integral over all displacements is one; this describes how the effect of neighbours attenuates with the displacement $\xi$. Second, $C(\xi)$ holds the pair density at displacement $\xi$; the integral thus adds up the effect of individuals at all displacements. Third, $\mathrm{d}^{\prime}$ is a parameter that scales the overall effect of these neighbours on the death rate.

Eq. (4) shows how the dynamics of the first moment are coupled to those of the second moment through $C(\xi)$. To close the dynamical system at order one, the mean-field assumption, $C(\xi)=N^{2}$, is traditionally used; this turns Eq. (4) into a closed dynamical system, familiar as the logistic equation (Pearl and Reed, 1920).

Extending the dynamics to the second moment gives

$$
\begin{aligned}
\dot{C}(\xi)= & 2 b m(\xi) N+b \int m\left(\xi^{\prime}\right) C\left(\xi+\xi^{\prime}\right) \mathrm{d} \xi^{\prime} \\
& +b \int m\left(\xi^{\prime}\right) C\left(-\xi+\xi^{\prime}\right) \mathrm{d} \xi^{\prime}-2 d C(\xi) \\
& -d^{\prime} \int w\left(\xi^{\prime}\right) T\left(\xi, \xi+\xi^{\prime}\right) \mathrm{d} \xi^{\prime} \\
& -d^{\prime} \int w\left(\xi^{\prime}\right) T\left(\xi, \xi^{\prime}\right) \mathrm{d} \xi^{\prime}-2 d^{\prime} w(\xi) C(\xi) .
\end{aligned}
$$

The second-order dynamics are more intricate because they have to account for all events that affect individuals in a pair. Despite the complexity, the only new ecological information here is a dispersal kernel $m(\xi)$ describing the probability density with which newborn offspring are displaced by an amount $\xi$ from their parents. Notice that, as in Eq. (4), these dynamics are coupled to those of the next spatial moment in the hierarchy through $T\left(\xi, \xi^{\prime}\right)$. To close the dynamical system at order two, a moment closure is needed that expresses $T$ in terms of $N$ and $C$. It is the appropriate choice of this closure that is the subject of this paper.

\section{Desirable qualities of moment closures}

Moment closures ultimately have to be selected based on their quantitative performance when describing specific spatial ecological dynamics. Yet there are a number of qualitative features that desirably a moment closure should possess. Since moment closures are approximations, it is immediately clear that not all such desiderata can be met by all possible closures. It is therefore instructive to pry out the advantages and shortcomings of moment-closure candidates based on a general analysis of these qualitative features (Section 4), before focusing on their quantitative performances in specific circumstances (Section 5). The present section serves to give an overview of the desiderata and to elucidate the logical relations between them.

\subsection{Qualities from definition of the third moment}

Positivity: Because the third moment describes the density of triplet configurations (and accordingly is obtained as a product of three densities all of which are non-negative, Eq. (3)) the third moment predicted by a closure, $\tilde{T}\left(\xi, \xi^{\prime}\right)$, must be non-negative for all $\xi$ and $\xi^{\prime}$,

D1: $\tilde{T}\left(\xi, \xi^{\prime}\right) \geqslant 0$.

As a product of three densities, valid moment closures must also furnish the predicted third moment with the cube of the physical dimension in which density is measured; for instance, in the case of a two-dimensional space, the dimension must be length ${ }^{-6}$.

Invariance under interchanging triplet members: It can be seen from Fig. 1 that, in a purely static and descriptive context, it is immaterial from which corner of the triangle the third moment is measured (three choices are possible). Moreover, the sequence in which the second and third member of a triplet are numbered is arbitrary (two choices possible). For this reason third moments possess a six-fold symmetry (D. Grey, pers. comm.). It would be desirable for third moments predicted by moment closures also to have this property, i.e.,

D2: $\tilde{T}\left(\xi, \xi^{\prime}\right)=\tilde{T}\left(\xi^{\prime}, \xi\right)=\tilde{T}\left(-\xi, \xi^{\prime}-\xi\right)=\tilde{T}\left(\xi^{\prime}-\xi,-\xi\right)=$ $\tilde{T}\left(-\xi^{\prime}, \xi-\xi^{\prime}\right)=\tilde{T}\left(\xi-\xi^{\prime},-\xi^{\prime}\right)$.

We return to this issue in the context of dynamics at the start of Section 4, where the corners do have distinct meanings as a result of modelling interactions.

\subsection{Qualities from averaging and large-distance limits}

Consistency under well-mixed conditions: When considering patterns in which the second moment is uniform, thus capturing no spatial structure whatsoever, a moment closure is not supposed to predict such structure in the third moment. We therefore want to ensure that

D3: $C=N^{2}$ implies $\tilde{T}=N^{3}$. 
A uniform second moment can arise, for instance, from Eqs. (4) and (5) when the neighbourhood sizes of competition and movement are made sufficiently large; alternatively, if individuals can move at times other than at birth, a population becomes well mixed and thus lacks any spatial structure if the rate of this movement is sufficiently large. Notice that relation D3 is presented for the limit of an infinite area, $A \rightarrow \infty$; otherwise the delta functions in Eqs. (2) and (3) that remove self-pairs cannot be neglected.

Mutual consistency of singlet and pair dynamics: Averaging a pair density $C(\xi)$ over all distances $\xi$ must obviously yield the total density of pairs, $A^{-1} \int C(\xi) \mathrm{d} \xi=N^{2}$ for $A \rightarrow \infty$; this follows from the definition of the pair density in Eq. (2). If pair densities settle on a constant value at large distances, the integral above implies that $\lim _{|\xi| \rightarrow \infty} C(\xi)=N^{2}$; this is simply because the integral is dominated by the asymptotic value (and the effect of self-pairs on this asymptotic value is negligible, since we have also to take the limit $A \rightarrow \infty)$. This in turn implies $\lim _{|\xi| \rightarrow \infty}(\mathrm{d} / \mathrm{d} t) C(\xi)=$ $2 N(\mathrm{~d} / \mathrm{d} t) N$. We can see that singlet and pair dynamics must thus be giving mutually consistent rates. Since the pair dynamics depend on the chosen moment closure, this consistency requirement imposes a constraint on feasible closures. It can be shown (Dieckmann and Law, 2000 , p. 439) that the consistency constraint is fulfilled if and only if

D4: $\lim _{|\xi| \rightarrow \infty} \tilde{T}\left(\xi, \xi^{\prime}\right)=N C\left(\xi^{\prime}\right)$.

Note that this constraint requires pair densities to tend to a constant value $\left(\lim _{|\xi| \rightarrow \infty} C(\xi)=N^{2}\right)$; a sufficient condition for this is the absence of large-scale spatial structure.

Integral constraints: Also, certain consistency conditions should apply when averaging triplet densities over one or both arguments. These conditions are again stated most conveniently for $A \rightarrow \infty$,

D5a: $A^{-1} \int \tilde{T}\left(\xi, \xi^{\prime}\right) \mathrm{d} \xi=N C\left(\xi^{\prime}\right)$, and

D5b: $A^{-1} \int \tilde{T}\left(\xi, \xi^{\prime}\right) \mathrm{d} \xi^{\prime}=N C(\xi)$.
These relations follow directly from the definition of triplet densities (Eq. (3)). Notice that, keeping in mind the integral constraint for pair densities, $A^{-1} \int C(\xi) \mathrm{d} \xi=N^{2}$, both D5a and D5b imply $A^{-2} \iint \tilde{T}\left(\xi, \xi^{\prime}\right) \mathrm{d} \xi \mathrm{d} \xi^{\prime}=N^{3}$

Asymptotic constraints: For triplet densities settling on a constant value at large distances, i.e. when the triplet density is constant in $\xi$ as $|\xi|$ becomes large and constant in $\xi^{\prime}$ as $\left|\xi^{\prime}\right|$ becomes large, constraints D5 above are fulfilled if and only if

D6a: $\lim _{|\xi| \rightarrow \infty} \tilde{T}\left(\xi, \xi^{\prime}\right)=N C\left(\xi^{\prime}\right)$, and

D6b: $\lim _{\left|\xi^{\prime}\right| \rightarrow \infty} \tilde{T}\left(\xi, \xi^{\prime}\right)=N C(\xi)$.

Notice that D6a turns out to be identical to D4. A sufficient condition for triplet densities to settle on constant values at large distances is the absence of largescale spatial structure. As in the case of the two integral constraints D5a and D5b, both D6a and D6b imply $\lim _{|\xi|,\left|\xi^{\prime}\right| \rightarrow \infty} \tilde{T}\left(\xi, \xi^{\prime}\right)=N^{3}$, given the asymptotic constraint for pair densities, $\lim _{|\xi| \rightarrow \infty} C(\xi)=N^{2}$.

\subsection{Qualities from dynamical invariance under relabelling}

A relabelling transformation amounts to partitioning, according to some recipe, the set of individuals of a single spatially distributed population into subsets by assigning a label (e.g. $1,2, \ldots$ ) to each individual. We refer to the original set of individuals as the 'total population' and to the newly created subsets as 'subpopulations'. Relabelling is the only alteration made (Fig. 2); it leaves the location of individuals unchanged, and the kernels and parameters of the subpopulations identical. Because the assigned labels have no bearing on the ecological dynamics (Arditi and Michalski, 1996),

$\mathrm{R}$ : The dynamics of the total population must be identical to those obtained by summing the dynamics of the subpopulations.

Hence we can ask to what extent approximate dynamics, based on particular moment closures, can
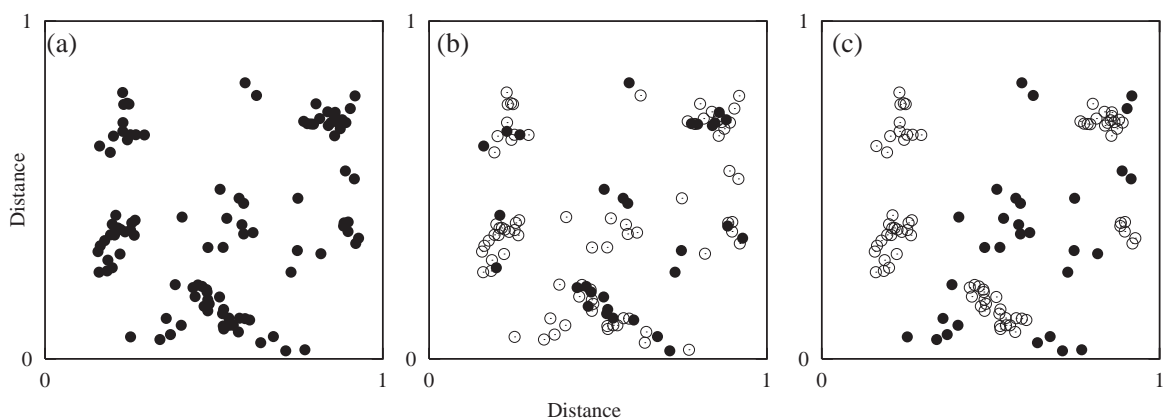

Fig. 2. Two relabelling transformations. (a) Total population prior to transformation. (b) Two subpopulations created by random relabelling of individuals into type 1 and type 2. (c) Two subpopulations created by non-random relabelling; individuals with fewer neighbours are more likely to be type 1 , and those with more neighbours are more likely to be type 2 . In (b) and (c), filled circles indicate individuals of type 1 , open circles those of type 2 . 
succeed in preserving this dynamical invariance under relabelling.

The simplest relabelling transformations are binary, dividing up the total population into two subpopulations, with individuals taking labels 1 or 2 . This entails the following relations between the singlet, pair, and triplet densities of the total populations and of the two subpopulations:

$$
\begin{aligned}
\mathrm{R} 1: N= & N_{1}+N_{2}, \\
\mathrm{R} 2: C & =C_{11}+C_{12}+C_{21}+C_{22}, \text { and } \\
\mathrm{R} 3: T= & T_{111}+T_{112}+T_{121}+T_{122} \\
& +T_{211}+T_{212}+T_{221}+T_{222} .
\end{aligned}
$$

Notice that R2 implies R1; this can be seen by integrating the terms in R2 with respect to $\xi$ using the relation $A^{-1} \int C_{i j}(\xi) \mathrm{d} \xi=N_{i} N_{j}$. In a similar way, R3 implies both R1 and R2; this is evident from integrating the terms in $\mathrm{R} 3$ as $A^{-1} \int T_{i j k}\left(\xi, \xi^{\prime}\right) \mathrm{d} \xi=N_{i} C_{j k}\left(\xi^{\prime}\right)$ and factorising the sum in terms of R1 and R2. Although we here focus on binary relabelling, any higher-order relabelling (using three or more different labels to define subpopulations) can be achieved through a sequence of binary relabelling transformations.

If the elementary interactions between individuals are pairwise, such as in the stochastic process on which Eqs. (4) and (5) are based, pair dynamics depend on triplet densities but not on any higher moments (see Discussion). Summing the pair dynamics of subpopulations, based on equations like (5), then shows that these dynamics are invariant under relabelling (in the sense of condition R) if and only if condition R3 is met. This means that any moment closure for triplet densities according to which R2 implies R3 ensures dynamical invariance under relabelling.

Invariance under random relabelling: Among the many relabelling recipes which could be used when testing for condition $\mathrm{R}$, the simplest is to assign a label $i=1,2$ to each individual with probability $p_{i}$, with $p_{1}+p_{2}=1$. We call this 'random relabelling' because labels are assigned to individuals irrespective of their neighbourhood structure and independently between individuals, as is illustrated in Fig. 2b. Random relabelling entails relations between the singlet, pair, and triplet densities of the total population and two subpopulations stricter than those given in conditions R1 to R3:

$$
\mathrm{R}^{\prime}: N_{i}=p_{i} N \text {, }
$$

$\mathrm{R} 2^{\prime}: C_{i j}=p_{i} p_{j} C$, and

$\mathrm{R} 3^{\prime}: T_{i j k}=p_{i} p_{j} p_{k} T$.

Evidently R1' implies R1, R2' implies R2, and R3 ${ }^{\prime}$ implies R3. Accordingly, moment closures $\tilde{T}$ that ensure dynamical invariance under random relabelling are characterized by the following condition:

D7a: $N_{i}=p_{i} N$ and $C_{i j}=p_{i} p_{j} C$ imply $\tilde{T}_{i j k}=p_{i} p_{j} p_{k} \tilde{T}$.

Invariance under non-random relabelling: Many other, non-random, relabelling schemes can be considered for which labelling depends on the local neighbourhood structure of individuals. For example, label 2 might be assigned to individuals that tend to experience more than average local competition, resulting from a high number and proximity of neighbouring conspecifics, with the remaining individuals receiving the label 1 ; this is illustrated in Fig. 2c. Under such a non-random scheme, conditions R2 ${ }^{\prime}$ and $\mathrm{R} 3^{\prime}$ would not generally hold with $p_{i}=N_{i} / N$. In particular, the pair densities $C_{11}, C_{12}=C_{21}$ and $C_{22}$ would exhibit differences beyond those captured by the label frequencies $p_{i}$, with $C_{11}$ showing a higher degree of short-range autocorrelation than $C$ and $C_{22}$ showing a corresponding lower degree. By contrast, conditions R1 to R3 can always be relied upon, even under non-random relabelling.

Since, up to third order, there are actually no logical constraints on non-random relabelling transformations other than R1 to R3, we can use these to specify the general condition for dynamical invariance under nonrandom relabelling as

D7b: $N=\sum_{i} N_{i}$ and $C=\sum_{i j} C_{i j}$ imply $\tilde{T}=\sum_{i j k} \tilde{T}_{i j k}$.

Here the sums extend over all labels considered, i.e. in the simplest case $i, j, k=1,2$.

Convergence of per capita growth rates under relabelling: Since the subpopulations generated by relabelling are ecologically equivalent by definition, their average per capita growth rates can only differ inasmuch as the subpopulations experience systematically different local neighbourhoods, as arising, for instance, from nonrandom relabelling. Since, again by definition, no dynamical forces exist that preserve these differences over the course of time, we should expect moment closures to reflect this feature by meeting the following qualitative condition:

D8: In the wake of a non-random relabelling transformation, per capita growth rates of the resulting subpopulations, as predicted by a moment closure $\tilde{T}$, should all equalize as time goes on.

\section{Properties of candidate closures}

To describe candidate closures for the third moment, it helps to have in mind the geometry of the locations of three individuals (Fig. 1); it is the density of a triplet of this kind that the third moment and its closures describe. In the dynamics, the corners of the triplet have distinct meanings and are not interchangeable. As we can see from Eq. (5), line 4, the individual at corner $k$, displaced from corner $i$ by $\xi^{\prime}$, serves as an 'actor' affecting the birth or death event at $i$ without itself being part of the focal pair $i j$, the density of which Eq. (5) describes. Accordingly, the individual at corner $i$ serves as a 'reactor', while the individual at corner $j$, displaced from corner $i$ by $\xi$, only serves as a 'watcher' of the interaction between individuals $i$ and $k$. Yet, individual $j$ is crucial: through the density of triplets it determines how likely 
Table 1

Summary of performance of power-1, power-2 and power-3 closures with respect to qualities D1 to D8 (defined in Section 3)

\begin{tabular}{llll}
\hline & Power-1 & Power-2 & Power-3 \\
\hline D1 & No under moderate & Yes, unless there is & Yes \\
& overdispersion & strong overdispersion & \\
D2 & Yes, if $\alpha=\beta=\gamma$ & Yes, if $\alpha=\beta=\gamma$ & Yes \\
D3 & Yes & Yes & Yes \\
D4 & Yes & Yes & Yes \\
D5a & Yes & Yes & Yes \\
D5b & Yes, if $\alpha=\beta=\gamma$ & Yes, if $\alpha=\beta=\gamma$ & Yes \\
D6a & Yes & Yes & Yes \\
D6b & Yes, if $\alpha=\beta=\gamma$ & Yes, if $\alpha=\beta=\gamma$ & Yes \\
D7a & Yes & Yes & Yes \\
D7b & Yes & Yes, except during an & No \\
& & initial transient & \\
D8 & Yes & Yes & No \\
\hline
\end{tabular}

individuals $k$ are to occupy positions at various locations around the focal pair $i j$.

This non-interchangeability of corners suggests that, within the context of Eq. (5), the symmetry D2 is not essential. With this in mind, we allow corners to have different weights where possible in the closures that follow. It is helpful to allow this extra flexibility because our experience has been that different weights can lead to quite different dynamics (see appendix of Law et al., 2003a, the online address of which is given in Law et al., 2003b, as well as in the list of references below). Removing the sixfold symmetry also means that D5b and D6b do not hold. Clearly, closures satisfying symmetry D2 are a special case of this more general weighting. It is interesting that the classical pair approximation, the closure assumption often used in lattice ecological models, ignores correlations in the $j k$ pair altogether (Sato and Iwasa, 2000; p. 346) and thus also does not satisfy D2.

Classes of closure below are called power-1, power-2, and power-3 according to the number of pair densities multiplied together (Dieckmann and Law, 2000; p. 441). The closures and their performance with respect to qualities D1 to D7 are described below (summarized in Table 1), and extend those given previously (Dieckmann and Law, 2000: 438 et seq.). We test D8 by numerical methods in Section 5.

\subsection{Power-1 closures}

Power-1 closures are suggested from an assumption, made on several occasions in the literature on moment dynamics, that the third central moment is zero (Bolker, 1999; Bolker and Pacala, 1997, 1999; Bolker et al., 2000). Power- 1 closures take as their building blocks just one pair density along an edge of the triplet and multiply it by the density at the opposite corner; this product ensures that the closure has the correct dimensions. The building blocks are summed and, if symmetry of the closure (D2) is not the overriding concern, they may have different weights, say $\alpha, \beta, \gamma$ for corners $i, j$, and $k$, respectively. To satisfy the conditions for averaging and large-distance limits (D3, D4, D5a, and D6a), an extra term needs to be introduced, giving the closure

$$
\begin{aligned}
\tilde{T}_{i j k}\left(\xi, \xi^{\prime}\right)= & \frac{1}{\beta}\left(\alpha N_{i} C_{j k}\left(\xi^{\prime}-\xi\right)+\beta N_{j} C_{i k}\left(\xi^{\prime}\right)\right. \\
& \left.+\gamma N_{k} C_{i j}(\xi)-(\alpha+\gamma) N_{i} N_{j} N_{k}\right) .
\end{aligned}
$$

This closure is fully invariant under relabelling and thus satisfies conditions D7a and D7b. However, the negative term in Eq. (6) is potentially rather large, and there is a danger of the closure becoming negative unless $\alpha$ and $\gamma$ are both small, thereby violating D1. (Fulfilling D3, D4, D5a, and D6a by multiplication rather than by subtraction might seem to avoid this problem (Keeling, 2000), but turns out to be impossible without upsetting the factorisation needed for D7b.) The completely symmetric case, $\alpha=\beta=\gamma$, is equivalent to assuming the central third moment is zero (Dieckmann and Law, 2000: 442).

\subsection{Power-2 closures}

Power-2 closures were originally suggested (Dieckmann and Law, 2000) in analogy to the classical pair approximation, used with some success as a closure in ecological lattice models (Sato and Iwasa, 2000). These closures take as their building blocks the product of two pair densities belonging to adjacent edges in the triangle $i j k$ and divide by the density at the corner the pairs have in common; again this ensures the closures have the correct dimensions. As before, if symmetry D2 is not the overriding concern, the closure can be a weighted sum of the building blocks, using weights $\alpha, \beta, \gamma$ for corners $i, j$, and $k$, respectively. To satisfy conditions D3, D4, D5a, and D6a, for averaging and large-distance limits, again an extra term needs to be subtracted, giving the closure

$$
\begin{aligned}
\tilde{T}_{i j k}\left(\xi, \xi^{\prime}\right)= & \frac{1}{\alpha+\gamma}\left(\alpha \frac{C_{i j}(\xi) C_{i k}\left(\xi^{\prime}\right)}{N_{i}}+\beta \frac{C_{i j}(\xi) C_{j k}\left(\xi^{\prime}-\xi\right)}{N_{j}}\right. \\
& \left.+\gamma \frac{C_{i k}\left(\xi^{\prime}\right) C_{j k}\left(\xi^{\prime}-\xi\right)}{N_{k}}-\beta N_{i} N_{j} N_{k}\right) .
\end{aligned}
$$

The negative term means that it is still possible for the closure to go negative, thereby violating D1; but, unlike in the power-1 closure, the negative term has only to balance one positive term.

While the closure above is invariant under random relabelling, and thus fulfils D7a, it does not meet the more stringent condition D7b for non-random relabelling 
unless the following discrepancy vanishes,

$$
\begin{aligned}
\frac{N}{N_{1} N_{2}} & \left(\sum_{i j k} \tilde{T}_{i j k}\left(\xi, \xi^{\prime}\right)-\tilde{T}\left(\xi, \xi^{\prime}\right)\right) \\
= & \alpha\left(\frac{C_{1}(\xi)}{N_{1}}-\frac{C_{2}(\xi)}{N_{2}}\right)\left(\frac{C_{1}\left(\xi^{\prime}\right)}{N_{1}}-\frac{C_{2}\left(\xi^{\prime}\right)}{N_{2}}\right) \\
& +\beta\left(\frac{C_{1}(-\xi)}{N_{1}}-\frac{C_{2}(-\xi)}{N_{2}}\right)\left(\frac{C_{1}\left(\xi^{\prime}-\xi\right)}{N_{1}}-\frac{C_{2}\left(\xi^{\prime}-\xi\right)}{N_{2}}\right) \\
& +\gamma\left(\frac{C_{1}(-\xi)}{N_{1}}-\frac{C_{2}(-\xi)}{N_{2}}\right) \\
& \times\left(\frac{C_{1}\left(-\xi^{\prime}+\xi\right)}{N_{1}}-\frac{C_{2}\left(-\xi^{\prime}+\xi\right)}{N_{2}}\right),
\end{aligned}
$$

where $C_{i}=C_{i 1}+C_{i 2}$. Notice that the terms on the righthand side are non-zero only when the two types have different per capita growth rates. This can be seen by, for instance, subtracting the per capita growth rate of type 2 from type 1 in Eq. (4) after relabelling; the difference is $\int w(\xi)\left(C_{1}(\xi) / N_{1}-C_{2}(\xi) / N_{2}\right) \mathrm{d} \xi$, which means that terms of the form $C_{1}(\xi) / N_{1}-C_{2}(\xi) / N_{2}$ must tend to zero as the per capita growth rates equalize. Thus, although there is an initial period during which D7b does not hold, the system moves to a state that satisfies D7b, as long as D8 is satisfied.

Weights for $\alpha, \beta$, and $\gamma$ considered in the literature include the completely symmetric case $(\alpha=\beta=\gamma$. Dieckmann and Law, 2000), a case in which the $i$-corner is given a higher weight $(\alpha=4, \beta=\gamma=1$ : Law et al., 2003a; Murrell and Law, 2003), and an extreme case of asymmetry $(\alpha=1, \beta=\gamma=0$ : Dieckmann and Law, 2000; Law and Dieckmann, 2000a) akin to the classical pair approximation. Another power-2 closure was used for animal movement in a heterogeneous landscape (Murrell and Law, 2000), but is not appropriate in a circumstance when there are births and deaths (i.e., when the first moment can change).

\subsection{Power-3 closure}

The power-3 closure has its roots in theoretical physics, where it is known as the Kirkwood superposition approximation (Kirkwood, 1935; see, e.g. Ziman, 1979). The closure takes the product of all three pair densities and divides by the product of densities at all three corners; again this ensures the closure has the correct dimensions. There is only one building block here, and therefore the symmetry condition D2 always applies. Conditions D3, D4, D5a, D5b, D6a, and D6b for averaging and large-distance limits are satisfied without any extra term, so we have simply

$\tilde{T}_{i j k}\left(\xi, \xi^{\prime}\right)=\frac{C_{i j}(\xi) C_{i k}\left(\xi^{\prime}\right) C_{j k}\left(\xi^{\prime}-\xi\right)}{N_{i} N_{j} N_{k}}$.

The absence of a correction term means that positivity D1 always applies. The above closure is invariant under random relabelling, D7a, but it does not pass the more stringent condition for non-random relabelling, i.e. D7b is not met.

\subsection{Comparison of candidate closures}

As summarized in Table 1, there is little to choose between the candidate closures as far as conditions D2 to D6 are concerned. Where the closures differ is in positivity (D1) and in invariance under relabelling (D7). These qualities are crucial: positivity because a change in sign would obviously play havoc with the signs of flux terms in the dynamics, and invariance because it would be illogical for the dynamics to be determined by changes that have no effect on properties of individuals. These two qualities in fact trade-off across closure power, power-1 closures doing best with respect to relabelling and worst with respect to positivity, the power- 3 closure doing the reverse, and power- 2 closures being intermediate.

\section{Numerical analysis of candidate closures}

To examine the closures further, two numerical analyses follow. The first compares the performance of closures in the short term following relabelling of populations close to equilibrium. The second compares the asymptotic state predicted by different closures in the long term. We used the spatial logistic model (4) and (5) for deterministic simulations, and a stochastic process of individual births, deaths, and movements for stochastic simulations, the stochastic process being the one on which Eqs. (4) and (5) are based (Law et al., 2003a). These simulations describe dynamics in a unit area, and use Gaussian competition and movement kernels

$w(\xi)=\frac{1}{W} \exp \left(-\frac{1}{2}|\xi|^{2} / s_{w}^{2}\right)$,

$m(\xi)=\frac{1}{M} \exp \left(-\frac{1}{2}|\xi|^{2} / s_{m}^{2}\right)$,

with parameters $s_{w}$ and $s_{m}$, respectively, describing the width of the kernels; $W$ and $M$ are normalization constants that give the right-hand sides a value 1 following integration over all displacements $\xi$.

\subsection{Equilibrium dynamics after relabelling}

A simple test for dynamical invariance under relabelling is to take a spatially distributed total population at equilibrium $\hat{N}, \hat{C}$, and split it up into two subpopulations by a relabelling transformation; 'equilibrium' of the total population here refers to the coupled system of both singlet and pair dynamics. Such relabelling should obviously still leave the total population at rest. 
The tests of dynamical invariance below used both random relabelling and non-random relabelling. To achieve random relabelling, we set initial conditions at $N_{1}=\hat{N}-50, N_{2}=50$, and $C_{i j}(\xi)=N_{i} N_{j} \hat{C}(\xi) / \hat{N}^{2}$. To achieve non-random relabelling, initial values for first moments were kept the same as those under random relabelling, whereas initial values for the second moments differed by subtraction of a term $0.5 N_{2}^{2} \exp (-5|\xi|) \hat{C}(\xi) / \hat{N}^{2}$ from $C_{12}=C_{21}$ and by redistributing this difference asymmetrically in the ratio $1: 3$ to $C_{11}$ and $C_{22}$, respectively.

According to R3, the closure obtained by summing the closures of the subpopulations should be the same as the closure of the total population. To test this, we considered a scaled deviation,

$\Delta(\xi)=\frac{\int w\left(\xi^{\prime}\right) \sum_{i j k}\left[\tilde{T}_{i j k}\left(\xi, \xi^{\prime}\right)-\tilde{T}\left(\xi, \xi^{\prime}\right)\right] \mathrm{d} \xi^{\prime}}{\int w\left(\xi^{\prime}\right) \tilde{T}\left(\xi, \xi^{\prime}\right) \mathrm{d} \xi^{\prime}}$,

at $\xi=0$ because this deviation is expected to be greatest at short displacements $\xi$. The effect of random relabelling is shown in Fig. 3a; as expected from R3', the deviation $\Delta(0)$ starts at zero for all three types of closure; however, as time goes on, the deviation remains at zero only for power- 1 and -2 closures, while a systematic discrepancy builds up in the power- 3 closure. The effect of non-random relabelling is shown in Fig. 3b: only the power- 1 closure is unaffected. The power- 2 closure has an initial discrepancy (see Eq. (8)), but this is small and quickly dies out, as the neighbourhoods of type- 1 and -2 individuals equalize. The power-3 closure has a larger initial discrepancy, and this grows as it does under random relabelling. Importantly, integration with the power-1 closure has to be stopped after only a short period of time because negative terms appear in the closure at short displacements (i.e. D1 is violated) due to the segregation in space of type- 1 and -2 individuals; this problem does not arise with the other closures.

According to D8, the per capita growth rates of the subpopulations should only differ insofar as relabelling causes the subpopulations to have different neighbourhoods, and these neighbourhoods should themselves equalize as time goes on. Thus, following random relabelling, where the neighbourhoods of the subpopulations start equal, the first moments $N_{1}, N_{2}$ of the subpopulations should be unchanged. (The total population is already at equilibrium with a per capita growth rate of zero, and this should also be true for subpopulations under random relabelling.) Fig. 3c shows that D8 holds for the power-1 and -2 closures, but not for the power-3 closure. The picture is similar following non-random relabelling (Fig. 3d), although there is now a period of adjustment of $N_{2}$ while the neighbourhoods equalize; after this $N_{2}$ behaves as it did under random relabelling.
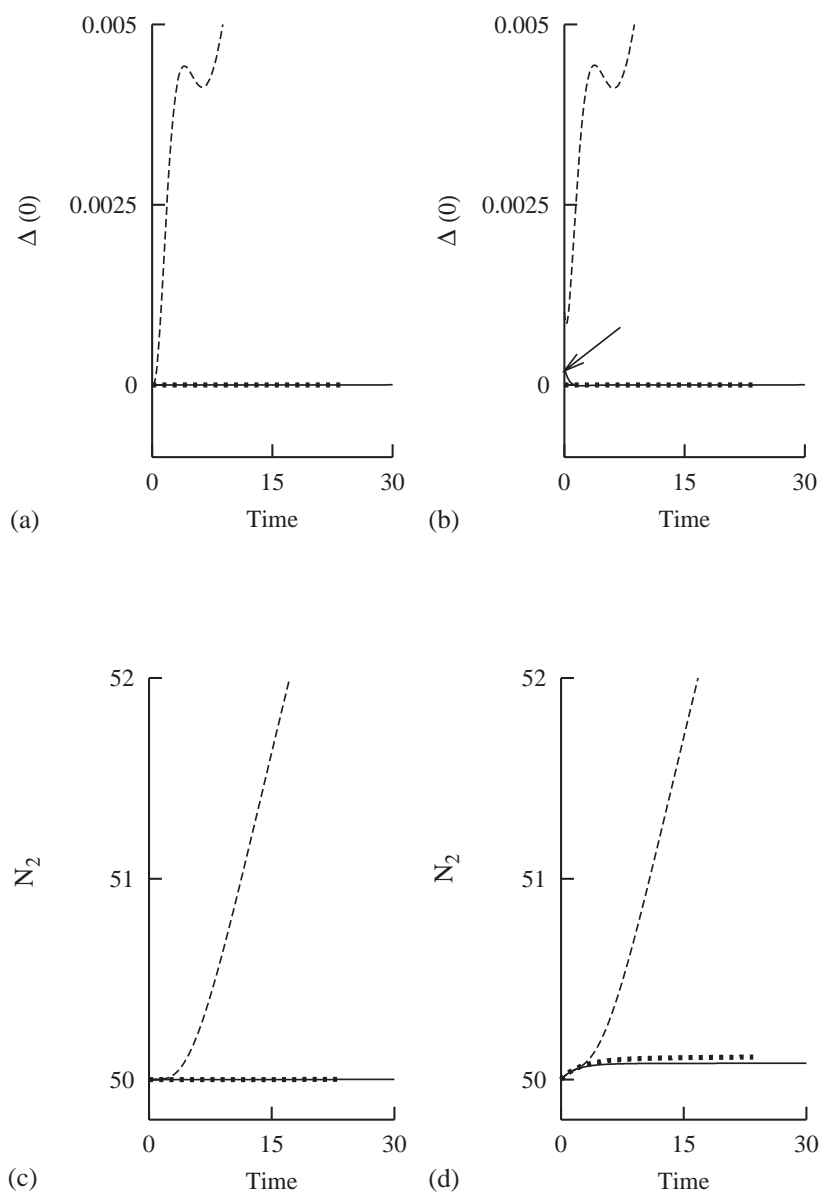

Fig. 3. Effect of relabelling transformations. Prior to relabelling, a numerical integration of Eqs. (4), (5) was carried out for 200 time units to get the first and second moments close to equilibrium. Fifty individuals per unit area were relabelled at time 0 , creating type 2; integration continued for a further 30 time units following relabelling. Scaled deviation $\Delta(\xi)$ at $\xi=0$ shown under (a) random relabelling, (b) non-random relabelling; the arrow points to the small initial deviation in the case of the power-2 closure. Population density $\mathrm{N}_{2}$ shown under (c) random relabelling, and (d) non-random relabelling. Symmetric power-1 closure: thick dotted lines; Symmetric power-2 closure: thin continuous lines; power-3 closure: thin dashed lines. Integration of the power- 1 closure was terminated when a closure value returned a negative value. Parameter values: $b_{1}=b_{2}=0.4$, $d_{1}=d_{2}=0.2, d_{11}^{\prime}=d_{22}^{\prime}=d_{21}^{\prime}=d_{12}^{\prime}=0.001, s_{w}=s_{m}=0.06$.

These results suggest that the power-3 closure is not altogether satisfactory as a closure of the hierarchy of equations. Moreover, although the power-1 closure works well under relabelling, it rather easily violates the condition of positivity. The power- 2 closure, being intermediate between the others, thus appears to be a reasonable compromise.

\subsection{Weights in power-1 and -2 closures}

There is still a question of how the weights $\omega=$ $\{\alpha, \beta, \gamma\}$, of power- 1 and -2 closures affect the accuracy of deterministic approximations to the stochastic 
processes. To gain insight into this, we computed the asymptotic value of the first moment under contrasting weights by numerical integration of Eqs. (4) and (5), and compared each with the corresponding moment averaged over some stochastic simulations.

In making this comparison, it is important to be aware that the closures may perform differently with different spatial structures. A good closure should be robust to change in spatial structure; for this reason we computed the asymptotic state across a range of spatial structures from strong aggregation to mild segregation (overdispersion) of individuals. This range was achieved by setting the parameters $s_{m}$ and $s_{w}$ to all $S=16$ pairwise combinations of the values $0.03,0.04,0.05,0.06$; the smallest values of $s_{m}$ give aggregation, and the smallest values of $s_{w}$ give overdispersion as long as $s_{m}$ is not too small (see Law et al., 2003a: Fig. 6b). For a given set of weights $\omega$, we determined the goodness-offit in terms of the following deviation measure

$D(\omega)=\frac{1}{S} \sum_{s} \frac{\left|\hat{N}(s, \omega)-\hat{N}_{i b}(s, \omega)\right|}{\hat{N}_{i b}(s, \omega)}$.

Here $\hat{N}(s, \omega)$ is the asymptotic value of the first moment obtained by integrating Eqs. (4) and (5) to steady state using the sth pair of kernel parameters values and weights $\omega$, and $\hat{N}_{i b}(s, \omega)$ is the corresponding value from the underlying individual-based, stochastic process, obtained as an average over 100 independent realizations. The summation over $s$ then adds up deviations across the range of spatial structures obtained with the different kernel parameters. For weights $\omega$, the deviation $D(\omega)$ thus measures the relative error in predicted first moments compared with actual first moments, averaged over a range of spatial structures; low deviations $D(\omega)$ indicate a close match.

Plotting this average deviation for each combination of weights reveals a surface with high and low values for both power-1 and power-2 closures (Fig. 4). Overall, power- 2 closures performed better than power-1 closures in two respects. First, the deviations between deterministic and stochastic simulations tended to be smaller. Second, in power- 1 closures it was always the case that the condition of positivity (D1) was violated for at least one combination of kernel parameters (such combinations were given a value of zero for the first moment). By contrast, this was never the case for the power-2 closures over the range of spatial structures investigated. Notice also that the denominator in the power-2 closure (Eq. (7)) leads to a singularity at just one point $\omega=\{0,1,0\}$, whereas it leads to a full line of singularities $\omega=\{\alpha, 0, \gamma\}$ in the case of power-1 closures (Eq. (6)).

The deterministic dynamics matched those of the stochastic process best if a low, but non-zero, value was given to $\beta$ (Fig. 4). The weights $\alpha$ and $\gamma$ were largely interchangeable, although the very lowest deviations were obtained by making either $\alpha$ large relative to $\gamma$ or $\gamma$ large relative to $\alpha$. (not visible at the resolution of Fig. 4). An asymmetric power-2 closure used previously with $\omega=\{4,1,1\}$ or, equivalently, $\omega=\left\{\frac{2}{3}, \frac{1}{6}, \frac{1}{6}\right\}$ (Law et al., 2003a; Murrell and Law, 2003) lies within the band of (a)

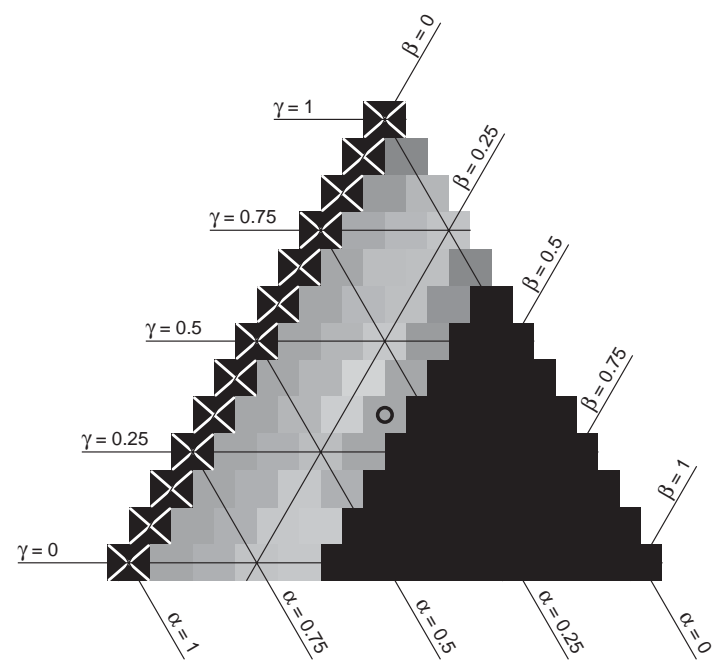

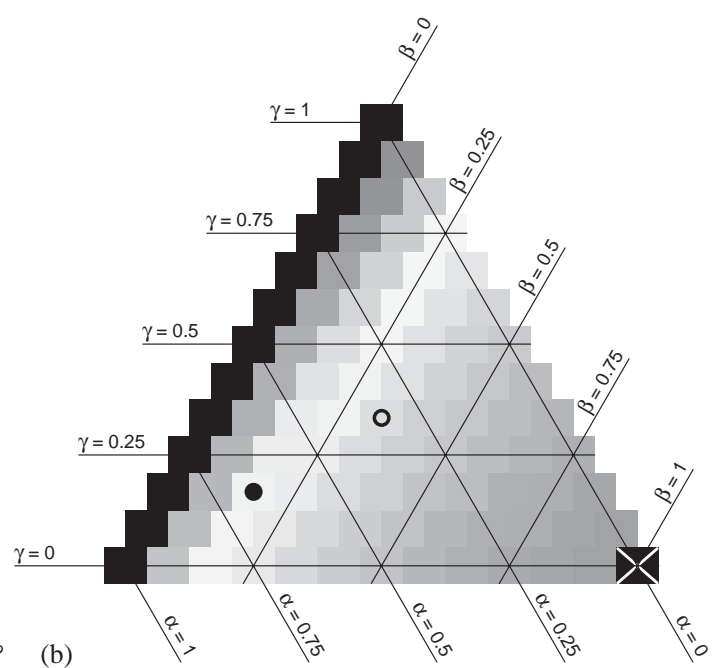

(b)

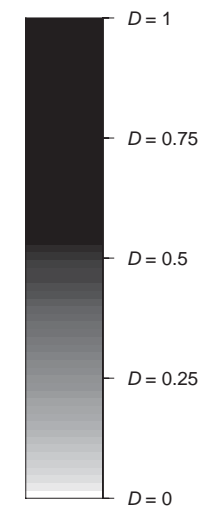

$D=0$

Fig. 4. Effect of weights $\alpha, \beta$, and $\gamma$ on the deviation between the equilibrium densities of a stochastic spatial logistic model and its deterministic approximation. (a) Power-1 closure. (b) Power-2 closure. Triangular graphs are used because weights multiplied by a common factor yield the same closure, so that only weights on the simplex $\alpha+\beta+\gamma=1$ need be considered. Each rectangle represents the value of the deviation $D(\omega)$ for a particular choice of $\omega=\{\alpha, \beta, \gamma\}$, with lighter shades representing a better match. Black squares with a white cross indicate infeasible weights that lead to a division by 0 . Open circles denote the point at which weights are symmetric; in (b), the special case $\omega=\{4,1,1\}$ previously used in the literature (equivalent to $\left\{\frac{2}{3}, \frac{1}{6}, \frac{1}{6}\right\}$ ) is denoted by a filled circle. Using $\omega=\{4,1,1\}$ reduces $D$ to 0.081 from $D=0.197$ for symmetric weights in the power- 2 closure. Note that $D=0.049$ for $\omega=\{1,3,8\}$ represents the minimum. Kernel parameter values are as described in the text; other parameter values as in Fig. 3. 
closest matches. The symmetric power-2 with $\omega=$ $\{1,1,1\}$ or, equivalently, $\omega=\left\{\frac{1}{3}, \frac{1}{3}, \frac{1}{3}\right\}$ (Dieckmann and Law, 2000) is also quite close to this region, whereas a fully asymmetric power- 2 closure with $\omega=\{1,0,0\}$ is clearly less satisfactory (Law and Dieckmann, 2000a).

\section{Discussion}

Many processes in ecology are local in space and cannot be captured by traditional mean-field models of population dynamics. It is therefore important to move on from the order-one closure of ecological dynamics (the mean-field assumption) at least to closures of order two (Dieckmann and Law, 2000; Filipe and Maule, 2003). Although several kinds of second-order closure have been suggested in earlier work, discrimination between them by formal methods has been difficult, because they satisfy most of the consistency conditions previously available. As a result, evaluation of closures has relied largely upon numerical comparisons of stochastic dynamics and deterministic dynamics that incorporate the closures (e.g. Dieckmann and Law, 2000; Filipe and Gibson, 2001; Filipe and Maule, 2003; Law et al., 2003a; van Baalen, 2000).

Here, by extending the toolbox of tests probing properties of third moments, we have been able to achieve some further discrimination between closures. Although some of the new properties, such as D5, do not improve matters, two criteria do help. The first is positivity, D1: because the third moment is a product of three densities, it should never be negative. The second is dynamical invariance under relabelling, D7: it would not make sense for the dynamics to be changed simply by relabelling identical individuals, as the underlying ecology remains unchanged. Power- $1,-2$, and -3 closures in fact trade-off these two properties, with the power- 2 closures falling into the middle-ground. Power- 2 closures remain positive except under very strong segregation (unpublished results); also, although non-random relabelling introduces an error in the case of power-2 closures, this error tends to zero as the neighbourhoods of the relabelled individuals equalize, at least in logistic and related models. Conversely, power-1 closures lose positivity under quite mild overdispersion, and the power-3 closure leads to a systematic discrepancy in the dynamics before and after relabelling. Power-2 closures thus have some merit as a reasonable compromise between positivity and invariance under relabelling, unless there are special arguments that call for power- 1 and -3 closures.

There is still a question as to how best to weight the building blocks of power-2 closures (or, when used, those of power-1 closures). The condition D2 for sixfold symmetry of the third moment calls for equal weighting, and it is certainly feasible to hold this in place. But, on the basis that the corners are not interchangeable in the dynamical system to which the moment closure is applied, some improvement in the approximation can be achieved by using different weights (Law et al., 2003a). Most critical is the weight $\beta$ relative to $\alpha+\gamma$. Weights $\alpha$ and $\gamma$ act in the same way in power- 1 and power-2 closures: either they both appear in the closure's negative term (power-1 closure, Eq. (6)), or neither of them does (power-2 closure, Eq. (7)), this distinguishes them from $\beta$. Thus the tendency of contours of the deviation measure shown in Fig. 4 to run approximately along lines of constant $\alpha+\gamma$ is not surprising; the smallest deviations require a relatively low value of $\beta$. Importantly, deviations are not minimized by the equal weights that would result in symmetric closures.

The problem of closure applies as much in discrete lattice space as it does in continuous space. In fact, the so-called classical pair-approximation (Matsuda et al., 1992; Sato and Iwasa, 2000) is a closure based on the $i$ corner of the triangle in Fig. 1 (Filipe and Maule, 2003), which motivated the fully asymmetric power- 2 closure introduced by Dieckmann and Law (2000). Other closures in discrete space have since been used (Filipe and Gibson, 2001; Filipe and Maule, 2003; Rand 1999; Sato and Iwasa, 2000; van Baalen, 2000), including a power- 2 closure that focuses on only the $j$-corner (Rand, 1999, p. 108), but nothing analogous to the general form of the power-2 closures in Eq. (7) has yet been considered. In view of the variable success of pair approximations (Filipe and Gibson, 2001; Filipe and Maule, 2003; van Baalen, 2000), it would be interesting to extend the results here to discrete space.

At the heart of the moment hierarchy described in Section 2 is an assumption that the dynamics of the $n$th moment depend on the $(n+1)$ th moment. It is important to realize that this is not a matter of mathematics: it is a biological assumption that individuals interact in pairs. The effect of neighbours on the fate of a single individual is assumed to be the sum of the interactions with each neighbour, and thus involves pair densities. The fate of a pair of individuals is the sum of the interactions the individuals in the pair have with each neighbour, and thus involves triplet densities, and so on. More generally, if the fate of an individual depends non-additively on $m$ other individuals, the dynamics of the $n$th moment depend on the $(n+m)$ th moment. The simple $n+1$ hierarchy is obviously much more tractable than the alternatives. Yet the fate of one individual can depend on more than one other individual, or on another individual and the physical environment. Dynamical systems could be devised for such interactions (Seabloom, Bolker and Bjørnstad, pers. comm.), but they would inevitably be much more intricate than those in which individuals interact in pairs. 
Ultimately, a second-order moment closure is an assumption, just as the traditional, first-order, meanfield closure is. The motivation for moving to secondorder closures is to link dynamics to spatial structure, a coupling that becomes important when individuals interact with close neighbours. However, truncation of the moment hierarchy at order two necessarily limits the types of spatial structure that can be successfully approximated. Spatial structures with significant amounts of information in spatial moments at higher orders obviously make order-two closures poor approximations. Thus caution is needed when using order-two closures; for instance, landscapes with spatial heterogeneity generated by topographic or anthropogenic factors could readily contain information at higher orders.

\section{Acknowledgements}

We thank B.M. Bolker, D. Grey, and V. Hutson for insightful discussions about moment closures. The Imperial College Parallel Computing Centre (ICPC) kindly donated computer processor time to generate Fig. 4. UD gratefully acknowledges financial support by the Austrian Science Fund; by the Austrian Federal Ministry of Education, Science, and Cultural Affairs; and by the European Research Training Network ModLife (Modern Life-History Theory and its Application to the Management of Natural Resources), funded through the Human Potential Programme of the European Commission.

\section{References}

Arditi, R., Michalski, J., 1996. Nonlinear food web models and their responses to increased basal productivity. In: Polis, G.A., Winemuller, K.O. (Eds.), Food Webs: Integration of Patterns and Dynamics. Kluwer, Boston, USA, pp. 122-133.

Bolker, B.M., 1999. Analytic models for the patchy spread of plant disease. Bull. Math. Biol. 61, 849-874.

Bolker, B.M., Pacala, S.W., 1997. Using moment equations to understand stochastically driven spatial pattern formation in ecological systems. Theor. Popul. Biol. 52, 179-197.

Bolker, B.M., Pacala, S.W., 1999. Spatial moment equations for plant competition: understanding spatial strategies and the advantages of short dispersal. Am. Nat. 153, 575-602.

Bolker, B.M., Pacala, S.W., Levin, S.A., 2000. Moment methods for ecological processes in continuous space. In: Dieckmann, U., Law, R., Metz, J.A.J. (Eds.), The Geometry of Ecological Interactions: Simplifying Spatial Complexity. Cambridge University Press, Cambridge, pp. 388-411.

Boots, M., Sasaki, A., 1999. 'Small worlds' and the evolution of virulence: infection occurs locally and at a distance. Proc. R. Soc. London B 266, 1933-1938.

Burrough, P.A., 1995. Spatial aspects of ecological data. In: Jongman, R.H.G., ter Braak, C.J.F., van Tongeren, O.F.R. (Eds.), Data Analysis in Community and Landscape Ecology. Cambridge University Press, Cambridge, pp. 213-251.
Dale, M.R.T., 1999. Spatial Pattern Analysis in Plant Ecology. Cambridge University Press, Cambridge.

Dieckmann, U., Law, R., 2000. Relaxation projections and the method of moments. In: Dieckmann, U., Law, R., Metz, J.A.J. (Eds.), The Geometry of Ecological Interactions: Simplifying Spatial Complexity. Cambridge University Press, Cambridge, pp. 412-455.

Dieckmann, U., Law, R., Metz, J.A.J., 2000. The Geometry of Ecological Interactions: Simplifying Spatial Complexity. Cambridge University Press, Cambridge.

Ellner, S.P., 2001. Pair approximation for lattice models with multiple interaction scales. J. Theor. Biol. 210, 435-447.

Filipe, J.A.N., Gibson, G.J., 2001. Comparing approximations to spatio-temporal models for epidemics with local spread. Bull. Math. Biol. 63, 603-624.

Filipe, J.A.N., Maule, M.M., 2003. Analytical methods for predicting the behaviour of population models with general spatial interactions. Math. Biosci. 183, 15-35.

Harada, Y., Ezoe, H., Iwasa, Y., Matsuda, H., Sato, K., 1995. Population persistence and spatially limited social interaction. Theor. Popul. Biol. 48, 65-91.

Hubbell, S.P., Ahumada, J.A., Condit, R., Foster, R.B., 2001. Local neighborhood effects on long-term survival of individual trees in a neotropical forest. Ecol. Res. 16, 859-875.

Keeling, M., 1999. Spatial models of interacting populations. In: McGlade, J. (Ed.), Advanced Ecological Theory: Principles and Applications. Blackwell Science, Oxford, pp. 64-99.

Keeling, M.J., 2000. Multiplicative moments and measures of persistence in ecology. J. Theor. Biol. 205, 269-281.

Kirkwood, J.G., 1935. Statistical mechanics of fluid mixtures. J. Chem. Phys. 3, 300-313.

Law, R., Dieckmann, U., 2000a. A dynamical system for neighbourhoods in plant communities. Ecology 81, 2137-2148.

Law, R., Dieckmann, U., 2000b. Moment approximations of individualbased models. In: Dieckmann, U., Law, R., Metz, J.A.J. (Eds.), The Geometry of Ecological Interactions: Simplifying Spatial Complexity. Cambridge University Press, Cambridge, pp. 252-270.

Law, R., Murrell, D.J., Dieckmann, U., 2003a. Population growth in space and time: spatial logistic equations. Ecology 84 , 252-262. Online appendix available in ESA's Electronic Data Archive as Ecological Archives E084-012-A1 at www.esapubs.org/ archive/ecol/E084/012/appendix-A.htm.

Law, R., Murrell, D.J., Dieckmann, U., 2003b. Population growth in space and time: spatial logistic equations (vol. 84, 252-262). Erratum. Ecology 84, 535.

Matsuda, H., Ogita, N., Sasaki, A., Sato, K., 1992. Statistical mechanics of population: the lattice Lotka-Volterra model. Prog. Theor. Phys. 88, 1035-1049.

Murrell, D.J., Law, R., 2000. Beetles in fragmented woodlands: a formal framework for dynamics of movement in ecological landscapes. J. Anim. Ecol. 69, 471-483.

Murrell, D.J., Law, R., 2003. Heteromyopia and the spatial coexistence of similar competitors. Ecol. Lett. 6, 48-59.

Norris, R.F., Elmore, C.L., Rejmánek, M., Akey, W.C., 2001. Spatial arrangement, density and competition between barnyardgrass and tomato: I. Crop growth and yield. Weed Sci. 49, 61-68.

Pacala, S.W., Levin, S.A., 1997. Biologically generated spatial pattern and the coexistence of competing species. In: Tilman, D., Kareiva, P. (Eds.), Spatial Ecology: The Role of Space in Population Dynamics and Interspecific Interactions. Princeton University Press, Princeton, New York, pp. 204-232.

Pearl, R., Reed, L.J., 1920. On the rate of growth of the population of the United States since 1790, and its mathematical representation. Proc. Natl Acad. Sci. USA 6, 275-288.

Rand, D.A., 1999. Correlation equations and pair approximations for spatial ecologies. In: McGlade, J.M. (Ed.), Advanced Ecological Theory. Blackwell Science, Oxford, pp. 100-142. 
Sato, K., Iwasa, Y., 2000. Pair approximations for lattice-based ecological models. In: Dieckmann, U., Law, R., Metz, J.A.J. (Eds.), The Geometry of Ecological Interactions: Simplifying Spatial Complexity. Cambridge University Press, Cambridge, pp. 341-358.

Sato, K., Matsuda, H., Sasaki, A., 1994. Pathogen invasion and host extinction in lattice structured populations. J. Math. Biol. 32, 251-268.

Stoll, P., Prati, D., 2001. Intraspecific aggregation alters competitive interactions in experimental plant communities. Ecology 82, 319-327.

Tilman, D., Karieva, P., 1997. Spatial Ecology: The Role of Space in Population Dynamics and Interspecific Interactions. Princeton University Press, Princeton. van Baalen, M., 2000. Pair approximations for different spatial geometries. In: Dieckmann, U., Law, R., Metz, J.A.J. (Eds.), The Geometry of Ecological Interactions: Simplifying Spatial Complexity. Cambridge University Press, Cambridge, pp. 359-387.

Wiegand, T., Moloney, K.A., 2004. Rings, circles, and null models for point pattern analysis in ecology. Oikos 104, 209-229.

Ziman, J.M., 1979. Models of Disorder: The Theoretical Physics of Homogeneously Disordered Systems. Cambridge University Press, Cambridge. 